\title{
Order parameter for ferromagnetic phase transition in the two-particle magnetic cluster system
}

\author{
N.A.Korynevskii ${ }^{1,2}$ \\ 1 Institute for Condensed Matter Physics \\ of the National Academy of Sciences of Ukraine, \\ 1 Svientsitskii Str., 79011 Lviv, Ukraine \\ 2 Institute of Physics, University of Szczecin, \\ 15 Wielkopolska Str., 70451 Szczecin, Poland
}

Received September 18, 2002

\begin{abstract}
Integration is performed over collective variables in the partition function functional of the two-particle magnetic cluster system using the fourth basic measure density. To rigorously consider the Gaussian and the nonGaussian fluctuations of the order parameter when dipole-dipole intercluster interactions take place, a two stage layer-by-layer method of integration is applied. To get the equation for the order parameter, the procedure of minimizing the integrand with the last (corresponding to zero values of quasimomentum and Matsubara's frequency) collective variable is used. Solutions of this equation in the phase transition point neighbourhood are found.
\end{abstract}

Key words: ferromagnets, cluster systems, functional, order parameter

PACS: $05.60 .+W, 05.70 . L n, 05.20 . D d, 52.25 . D g, 52.25 . F i$

\section{Introduction}

As it is well known the first successful theory for cooperative phenomena, namely for ferromagnetic phase transition description, was introduced by P.Weiss in his molecular field theory [1]. In this theory the only atom is taken into consideration, all interactions of this atom with the other atoms of the crystal being replaced due to the action of the effective (molecular) field. The role and the significance of this theory in the further development of the phase transition theory is enormous, but it is largely insufficient as well.

Thought the theory distinctly describes the main fundamental physical properties of ferromagnets it does not give a sufficiently good quantitative agreement with the experiment. This is caused by a complete neglect of correlation effects between 
particles. Therefore, it is only natural to make at least a small region of the crystal undergo a rigorous treatment and to replace the effect of the remaining regions of the crystal by the self-consistent (i.e., molecular) field.

The introduction of this idea into the theory of ferromagnetism is connected with T.Oguchi, H.A.Bethe, R.Peierls, R.Kikuchi, P.Weiss and others [2]. In this approach, the main attention is focused on the arbitrarily chosen central atom and its $z$ nearest neighbours. The application of statistical physics methods to the system of $z+1$ atoms (cluster) in the molecular field makes it possible to calculate all thermodynamic functions and to investigate their behaviour in a wide temperature range including the phase transition point. Hence, one may find a better coincidence of the physical values predicted in this approximation with the experiment as compared with the same for the simple molecular field approximation. The main difference of theoretical and experimental dependencies is concentrated both near the phase transition point and at very low temperatures, where quantum properties of the investigated system play an essential role. Since the cluster approximation is only an improved version of the ordinary molecular field theory and correlation effects in this approach are completely ignored, the critical behaviour of thermodynamic functions near $T_{\mathrm{c}}$ is far from being true. However, for the systems possessing a narrow phase transition point neighbourhood the application of the cluster approach is well founded, with the exception of low temperatures (in the quantum systems case).

For ferroelectric systems, the cluster approximation has been applied by R. Blinc [3]. The starting-point in his investigations of a ferroelectric phase transition in KDP was the Kubo cluster cumulant expansion [4]. Having confined himself to the first order of expansion, R.Bline has found an expression for free energy of the system based on the principle of consistency for many-particle matrices of density. The paper [3] appears to be a foundation for the subsequent investigations of hydrogen bonded ferroelectrics of KDP-type [5].

The cluster approximation in the form based on the paper [3] was used by R.R.Levitskii with collaborators in the numerous investigations concerning KDP and isomorphous crystals with hydrogen bounds [6-16]. The scope of his interests is very wide, i.e., from static properties to the dynamics and relaxation behaviour of the systems. From mathematical point of view, the problem is how to simultaneously take into account long and short range interparticle interactions. The cluster approximation for short range forces and the molecular field for long range forces of interparticle interactions is a good approach for the crystals with a small critical region. The main achievements herein appear to be as follows: a quantitative description of polarization and phase transition temperature, tensor of static and dynamic dielectric susceptibility, times of relaxation, the effect of stress on the physical properties for different hydrogen bonded ferroelectrics. These results are realistic due to a scrupulous selection of the basic parameters for the Hamiltonian of interparticle interactions in the crystals.

Notwithstanding the great advantages of the cluster approximation method (i.e., a clear physical idea, fairly simple mathematical form and the possibility to obtain 
good coincidence with experimental facts), the method is completely unacceptable in the vicinity of the phase transition point neighbourhood. For this kind of investigations, we need the methods taking into consideration the Gaussian and non-Gaussian fluctuations of the order parameter. In the present paper, the collective variables method [17] is used for detailed investigations near the phase transition point of ferromagnetic system with the cluster type of short-range interparticle interaction. In [18], the functional representation was obtained for a partition function functional of the system containing an exchange Heisenberg-type interaction between the spins from the same cluster and magnetic dipole interaction of spins from different clusters. The magnetic dipole interaction is decisive in a ferromagnetic phase transition, which takes place in those systems. For example, in the $\mathrm{Cu}_{2}\left(\mathrm{CH}_{3} \mathrm{CO}_{2}\right)_{4}$. $2 \mathrm{H}_{2} \mathrm{O}$ crystal, the unicomponent order parameter describing magnetization along the ferromagnetic axis is completely determined by the magnetic dipole potential of interaction between $z$-components of the total spin of the clusters [2].

The idea of the basic form of a short range cluster-type interaction in the partition function functional is very close to the cluster approximation method exploited by R.R.Levitskii, but we do not introduce any internal field (i.e., molecular field) which is usually determined by minimization procedure. Such a field is proportional to the order parameter of the system investigated and must be obtained due to a rigorous procedure of integration over collective variables in the functional.

Taking into account the expressions for cluster cumulants of the two-particle cluster system with dipole-dipole intercluster interactions of the Ising-type [18], based on the general form of partition function functional $[17,18]$ we have:

$$
\begin{aligned}
& Z=Z_{0} \int\left(\mathrm{d} \rho_{\lambda}(\vec{k}, \nu)\right)^{N} \exp \left\{\sum_{\lambda=7,16} \sum_{k, \nu} \frac{\beta}{2} \Phi_{\lambda}(\vec{k}) \rho_{\lambda}(\vec{k}, \nu) \rho_{\lambda}(-\vec{k},-\nu)\right\} \\
& \times \int\left(\mathrm{d} \omega_{\lambda}(\vec{k}, \nu)\right)^{N} \exp \left\{\mathrm{i} 2 \pi \sum_{\lambda=7,16} \sum_{k, \nu}\left[\rho_{\lambda}(\vec{k}, \nu)-\mathcal{M}_{\lambda}(\vec{k}, \nu) \delta_{\lambda 16}\right] \omega_{\lambda}(\vec{k}, \nu)\right. \\
&-\frac{(2 \pi)^{2}}{2} \sum_{\lambda=7,16} \sum_{k, \nu} \mathcal{M}_{\lambda \lambda}(\vec{k}, \nu,-\vec{k},-\nu) \omega_{\lambda}(\vec{k}, \nu) \omega_{\lambda}(-\vec{k},-\nu) \\
&+\frac{(\mathrm{i} 2 \pi)^{3}}{3 !} \sum_{\lambda_{1}, \lambda_{2}=7,16} \sum_{\substack{k_{1}, k_{2}, k_{3} \\
\nu_{1}, \nu_{2}, \nu_{3}}} \mathcal{M}_{\lambda_{1} \lambda_{1} \lambda_{2}}\left(\vec{k}_{1}, \nu_{1}, \vec{k}_{2}, \nu_{2}, \vec{k}_{3}, \nu_{3}\right) \\
&+ \frac{(2 \pi)^{4}}{4 !} \sum_{\lambda_{1}, \lambda_{2}=7,16} \sum_{\substack{k_{1}, k_{2}, k_{3}, k_{4} \\
\nu_{1}, \nu_{2}, \nu_{3}, \nu_{4}}} \mathcal{M}_{\lambda_{1} \lambda_{1} \lambda_{2} \lambda_{2}}\left(\vec{k}_{1}, \nu_{1}, \vec{k}_{2}, \nu_{2}, \vec{k}_{3}, \nu_{3}, \vec{k}_{4}, \nu_{4}\right) \\
& \times\left[\delta_{\lambda_{1} 7} \delta_{\lambda_{2} 7}+\delta_{\lambda_{1} 7} \delta_{\lambda_{2} 16}+\delta_{\lambda_{1} 16} \delta_{\lambda_{2} 16}\right] \\
&\left.\times \omega_{\lambda_{1} 16}\left(\vec{k}_{1}, \nu_{1}\right) \omega_{\lambda_{1}}\left(\vec{k}_{2}, \nu_{2}\right) \omega_{\lambda_{2}}\left(\vec{k}_{3}, \nu_{3}\right) \omega_{\lambda_{2}}\left(\vec{k}_{4}, \nu_{4}\right)\right\} \\
&\left.Z_{0}=\vec{k}_{1}, \nu_{1}\right) \omega_{\lambda_{1}}\left(\vec{k}_{2}, \nu_{2}\right) \omega_{\lambda_{2}}\left(\vec{k}_{3}, \nu_{3}\right) \\
& Z_{01}^{N}=\left[\mathrm{e}^{\frac{\beta V}{2}}(1+2 \cosh \beta h)+\mathrm{e}^{-\frac{3 \beta V}{2}}\right]^{N} \cdot
\end{aligned}
$$


Here $\rho_{\lambda}(\vec{k}, \nu)$ is a collective variable in the frequency-momentum representation; $\omega_{\lambda}(\vec{k}, \nu)$ is a value conjugated to $\rho_{\lambda}(\vec{k}, \nu) ; \beta=1 / k T, k$ is the Boltzmann constant, $T$ is the absolute temperature; $h$ is an external magnetic field, $V$ is the energy of two-particle cluster;

$$
\nu=\frac{2 \pi}{\beta} n, \quad n=0,1,2, \ldots
$$

is the Matsubara's frequency.

For a Fourier transform of the intercluster dipole-dipole potential responsible for ferromagnetic ordering of spins we shall use an expression

$$
\Phi_{16}(\vec{k})=\varphi_{0}-\lambda \cos ^{2} \theta-A|\vec{k}|^{2},
$$

where $\theta$ is a polar angle in the ellipsoid of rotation coordinate system, $\varphi_{0}, \lambda, A$ are constants which may be found, for example, using the Ewald method [19]. Out of two collective modes corresponding to the variables $\rho_{7}(\vec{k}, \nu)$ and $\rho_{16}(\vec{k}, \nu)$ the most significant in the ferromagnetic phase transition is the mode connected with $\rho_{16}(\vec{k}, \nu)$. Specifically, the mean value of $\rho_{16}(\vec{k}, \nu)$ forms the order parameter of the system investigated [18]:

$$
M\left(\vec{R}_{q}\right)=\left\langle\sigma_{1}^{z}\left(\vec{R}_{q}\right)+\sigma_{2}^{z}\left(\vec{R}_{q}\right)\right\rangle=\sqrt{2}\left\langle\rho_{16}(\vec{k}, \nu)\right\rangle
$$

where $\sigma_{f}^{z}\left(\vec{R}_{q}\right)$ is the spin of $f$-th particle in the $q$ cluster.

In order to obtain $M\left(\vec{R}_{q}\right)$ (for ferromagnetic ordering $M\left(\vec{R}_{q}\right)=M$ ) the integration of (1.1) over all variables with the exception of $\rho_{16}(0,0)$ is necessary. The present paper is carried out with the purpose of obtaining the equation for $M$ and analysing its solutions.

\section{Integration over collective variables $\rho_{7}(\vec{k}, \nu)$}

As it was mentioned above, the collective variables $\rho_{7}(\vec{k}, \nu)$ did not take immediate part in the ferromagnetic order parameter formation. Hence, they are not directly connected with the collective mode which undergoes a peculiarity in the phase transition point. The shape of distribution curve of $\rho_{7}(\vec{k}, \nu)$ variables is unimportant with respect to temperature change (it remains Gaussian when temperature passes $\left.T_{\mathrm{c}}\right)$. Thus, for rigorous integration over $\rho_{7}(\vec{k}, \nu)$ in $(1.1)$, the Gaussian measure density must be used. The higher orders of $\rho_{7}(\vec{k}, \nu)$ and $\omega_{7}(\vec{k}, \nu)$ variables products in (1.1) must be decomposed into a series and every addendum should be represented as a Gaussian momentum. Proceeding from the expressions for the cluster cumulants of the investigated system [18] one may find that for weak external fields and for temperatures close to characteristic ones, for example, for $\mathrm{Cu}_{2}\left(\mathrm{CH}_{3} \mathrm{CO}_{2}\right)_{4}$. $2 \mathrm{H}_{2} \mathrm{O}\left(T_{\mathrm{c}}=270 \mathrm{~K}, V=800 \mathrm{~K}\right)[2]$, the main field dependence is connected only with $\rho_{16}(\vec{k}, \nu)$ component:

$$
\mathcal{M}_{16} \approx 0,47 \beta h, \quad \mathcal{M}_{771616} \approx 0,026 \beta h, \quad \mathcal{M}_{161616} \approx 0,21(\beta h)^{3} .
$$


Using linear approximation in $\beta h$ and taking into account the relation $\beta h / V \ll 1$ we will take only first, second and fourth power of variables $\omega_{\lambda}(\vec{k}, \nu)$ in the form (1.1). It may be noted that the third-order cumulants are omitted because their effect on the measure density is small. An exact removal of these cumulants can be performed by "shifting" the procedure for $\omega_{\lambda}(\vec{k}, \nu)$ the variables (see [20]). In the present paper we will use the "shifting" procedure only for the variables with $\lambda=16$.

After expanding the exponents with $\mathcal{M}_{7777}$ and $\mathcal{M}_{771616}$ into a series and using the variables $\omega_{\lambda}(\vec{k}, \nu)$ for the $n$-order product, the $n$-order derivation will be as follows:

$$
\begin{aligned}
& \text { const } \exp \left\{\mathrm{i} 2 \pi \sum_{k, \nu} \rho_{\lambda}(\vec{k}, \nu) \omega_{\lambda}(\vec{k}, \nu)\right. \\
&\left.\quad-\frac{(2 \pi)^{2}}{2} \sum_{k, \nu} \mathcal{M}_{\lambda \lambda}(\vec{k}, \nu,-\vec{k},-\nu) \omega_{\lambda}(\vec{k}, \nu) \omega_{\lambda}(-\vec{k},-\nu)\right\} \\
& \times \omega_{\lambda}\left(\vec{k}_{1}, \nu_{1}\right) \omega_{\lambda}\left(\vec{k}_{2}, \nu_{2}\right) \ldots \omega_{\lambda}\left(\vec{k}_{n}, \nu_{n}\right)= \\
&= \frac{\partial^{n}}{(2 \pi \mathrm{i})^{n}} \cdot \frac{\partial^{n}}{\partial \rho_{\lambda}\left(\vec{k}_{1}, \nu_{1}\right) \partial \rho_{\lambda}\left(\vec{k}_{2}, \nu_{2}\right) \ldots \partial \rho_{\lambda}\left(\vec{k}_{n}, \nu_{n}\right)} \\
& \exp \left\{\mathrm{i} 2 \pi \sum_{k, \nu} \rho_{\lambda}(\vec{k}, \nu) \omega_{\lambda}(\vec{k}, \nu)\right. \\
&\left.\quad-\frac{(2 \pi)^{2}}{2} \sum_{k, \nu} \mathcal{M}_{\lambda \lambda}(\vec{k}, \nu,-\vec{k},-\nu) \omega_{\lambda}(\vec{k}, \nu) \omega_{\lambda}(-\vec{k},-\nu)\right\}
\end{aligned}
$$

we will integrate $(1.1)$ over the variables $\omega_{7}(0,0), \omega_{7}^{c}(\vec{k}, \nu), \omega_{7}^{s}(\vec{k}, \nu)$, where

$$
\omega_{\lambda}(\vec{k}, \nu)=\frac{1}{2}\left[\omega_{\lambda}^{c}(\vec{k}, \nu)-\mathrm{i} \omega_{\lambda}^{s}(\vec{k}, \nu)\right] .
$$

Formula $(2.2)$ reduces the integration over $\omega_{7}(\vec{k}, \nu)$ to the calculation of a simple integral:

$$
\begin{aligned}
I= & \int\left(\mathrm{d} \omega_{7}(\vec{k}, \nu)\right) \exp \left\{\mathrm{i} 2 \pi \sum_{k, \nu} \rho_{7}(\vec{k}, \nu) \omega_{7}(\vec{k}, \nu)\right. \\
& \left.-\frac{(2 \pi)^{2}}{2} \sum_{k, \nu} \mathcal{M}_{77}(\vec{k}, \nu,-\vec{k},-\nu) \omega_{7}(\vec{k}, \nu) \omega_{7}(-\vec{k},-\nu)\right\}
\end{aligned}
$$

which is equal to

$$
I=\left\{\prod_{k, \nu} \frac{1}{\sqrt{\pi \mathcal{M}_{77}(0, \nu)}} \frac{1}{\pi \mathcal{M}_{77}(\vec{k}, \nu)}\right\} \exp \left\{-\frac{1}{2} \sum_{k, \nu} \frac{\rho_{7}(\vec{k}, \nu) \rho_{7}(-\vec{k},-\nu)}{\mathcal{M}_{77}(\vec{k}, \nu)}\right\} .
$$

Now, the integration of (1.1) over $\rho_{7}(0,0), \rho_{7}^{c}(\vec{k}, \nu), \rho_{7}^{s}(\vec{k}, \nu)$, where

$$
\rho_{\lambda}(\vec{k}, \nu)=\rho_{\lambda}^{c}(\vec{k}, \nu)+\mathrm{i} \rho_{\lambda}^{s}(\vec{k}, \nu)
$$


may be carried out. Hence, after summing up the infinity series of screening potential

$$
g_{7}(\vec{k}, \nu)=\frac{\beta \Phi_{7}(\vec{k})}{1-\beta \Phi_{7}(\vec{k}) \mathcal{M}_{77}(k, \nu)},
$$

we obtained, for the total partition function functional (1.1), the functional integral over exclusively collective variables $\rho_{16}(\vec{k}, \nu)$ which form the branch of collective vibration of the system active in the phase transition:

$$
\begin{aligned}
Z= & Z_{0} Z_{7} \int\left(\mathrm{d} \rho_{16}(\vec{k}, \nu)\right)^{N} \exp \left\{\sum_{k, \nu} \frac{\beta}{2} \Phi_{16}(\vec{k}) \rho_{16}(\vec{k}, \nu) \rho_{16}(-\vec{k},-\nu)\right\} \\
& \times \int\left(\mathrm{d} \omega_{16}(\vec{k}, \nu)\right)^{N} \exp \left\{\mathrm{i} 2 \pi \sum_{k, \nu}\left[\rho_{16}(\vec{k}, \nu)-\mathcal{M}_{16}(\vec{k}, \nu) \delta(\vec{k}) \delta(\nu)\right] \omega_{16}(\vec{k}, \nu)\right. \\
& -\frac{(2 \pi)^{2}}{2} \sum_{k, \nu}\left[\mathcal{M}_{1616}(\vec{k}, \nu,-\vec{k},-\nu)\right. \\
& \left.+\frac{1}{12} \sum_{k^{\prime}, \nu^{\prime}} \mathcal{M}_{771616}\left(\vec{k}^{\prime}, \nu^{\prime},-\vec{k}^{\prime},-\nu^{\prime}\right) g_{7}\left(\vec{k}^{\prime}, \nu^{\prime}\right)\right] \omega_{16}(\vec{k}, \nu) \omega_{16}(-\vec{k},-\nu) \\
& +\frac{(2 \pi)^{4}}{4 !} \sum_{\substack{k_{1}, k_{2}, k_{3}, k_{4} \\
\nu_{1}, \nu_{2}, \nu_{3}, \nu_{4}}} \mathcal{M}_{16161616}\left(\vec{k}_{1}, \nu_{1}, \vec{k}_{2}, \nu_{2}, \vec{k}_{3}, \nu_{3}, \vec{k}_{4}, \nu_{4}\right) \\
& \left.\times \omega_{16}\left(\vec{k}_{1}, \nu_{1}\right) \omega_{16}\left(\vec{k}_{2}, \nu_{2}\right) \omega_{16}\left(\vec{k}_{3}, \nu_{3}\right) \omega_{16}\left(\vec{k}_{4}, \nu_{4}\right)\right\} \\
Z_{7}= & \prod_{k, \nu}\left\{\frac{1}{\sqrt{1-\beta \Phi_{7}(0) \mathcal{M}_{77}(0,0)}} \cdot \frac{1}{1-\beta \Phi_{7}(\vec{k}) \mathcal{M}_{77}(\vec{k}, \nu)}\right\} \\
& \times \exp \left\{\frac{1}{8} \sum_{\substack{k, \nu \\
k^{\prime}, \nu^{\prime}}} \mathcal{M}_{7777}\left(\vec{k}, \nu,-\vec{k},-\nu, \vec{k}^{\prime}, \nu^{\prime},-\vec{k}^{\prime},-\nu^{\prime}\right) g_{7}(\vec{k}, \nu) g_{7}\left(\vec{k}^{\prime}, \nu^{\prime}\right)\right\}
\end{aligned}
$$

The term in front of the integral in (2.8) at temperatures close to a ferromagnetic phase transition point is continuous, all critical peculiarities of the system been contained in the functional integral over $\rho_{16}(\vec{k}, \nu)$. In the simplest Gaussian approximation $T_{\mathrm{c}}$ is a solution of the equation:

$$
1-\beta_{\mathrm{c}} \Phi_{16}(0) \tilde{\mathcal{M}}_{1616}(0,0)=0,
$$

where $\tilde{\mathcal{M}}_{1616}(0,0)$ is a renormalized second cumulant at $\vec{k}=0$ and $\nu=0$. The complete expression for $\tilde{\mathcal{M}}_{1616}(\vec{k}, \nu)$ has been obtained after summation over $\nu^{\prime}$ :

$$
\begin{aligned}
& \tilde{\mathcal{M}}_{1616}(\vec{k}, \nu)=\mathcal{M}_{1616}(\vec{k}, \nu) \\
& -\frac{M}{24} \sum_{k^{\prime}} \beta \Phi_{7}\left(\vec{k}^{\prime}\right)\left[\frac{1}{V-\beta \Phi_{7}\left(\vec{k}^{\prime}\right) N}+\frac{\beta \sqrt{V} \operatorname{coth} \beta \sqrt{V\left(V-\beta \Phi_{7}\left(\vec{k}^{\prime}\right) N\right.}}{\sqrt{V-\beta \Phi_{7}\left(\vec{k}^{\prime}\right) N}}\right], \\
& M=\frac{\sinh \beta V}{\beta Z_{01}^{2}}\left(\cosh \beta h-\frac{2 \mathrm{e}^{\frac{\beta V}{2}} \sinh ^{2} \beta h}{Z_{0}}\right), \quad N=\frac{\mathrm{e}^{-\frac{\beta V}{2}} \sinh \beta V}{\beta Z_{01}} .
\end{aligned}
$$


When short-range forces are absent $\left(V=0, \mathcal{M}_{77}=\mathcal{M}_{1616}=1 / 4, \mathcal{M}_{7777}=\right.$ $\mathcal{M}_{771616}=\mathcal{M}_{16161616}=-1 / 16, \Phi_{16}=4 J_{11}, \Phi_{7}=0, \mathcal{M}_{\lambda \ldots \lambda}=\mathcal{M}_{\lambda \ldots \lambda}(0,0), \Phi_{\lambda}=$ $\Phi_{\lambda}(0), J_{11}$ is a pair long-range interparticle potential at $\left.\vec{k}=0\right)$, the equation $(2.9)$ reduces to the expression for the ferromagnetic phase transition temperature in the one-sublattice crystal (in Gaussian, or the so-called, random phase approximation):

$$
T_{\mathrm{c}}=J_{11} .
$$

It is well-known that formula (2.12) gives a somewhat big value for $T_{\mathrm{c}}$. Hence, for a precise calculation of $T_{\mathrm{c}}$, the higher order approximation (taking into account the non-Gaussian distribution of collective variables in (1.1)) should be used. We will exploit this approximation to integrate $(1.1)$ over $\rho_{16}(\vec{k}, \nu)$ variables.

\section{Integration over $\rho_{16}(\vec{k}, \nu)$ collective variables}

Since the order parameter, which arises below a ferromagnetic phase transition point, is determined by the (1.4) formula, integration of (2.8) functional over all variables with the exception of the variable $\rho_{\lambda}(0,0)$ is necessary. But it may be checked (see [18]), that cumulants $\tilde{\mathcal{M}}_{1616}, \mathcal{M}_{16161616}$ are independent of Matsubara frequency. Because the phase transition in its origin is the classical phenomenon (it corresponds to $\nu=0$ ) and the spectrum of $\nu$ at real finite values of $T$ is discrete (see (1.2)), only the variables

$$
\rho_{k}=\rho_{16}(\vec{k}, 0)
$$

are the most important ones in the functional (2.8).

The integrand in (2.8) reduces to the multiproduct form when a transition to the knot variables has been performed:

$$
\begin{gathered}
\rho_{l}=\frac{1}{\sqrt{N}} \sum_{k} \rho_{k} \mathrm{e}^{\mathrm{i} \vec{k} \vec{l}} \\
\omega_{l}=\frac{1}{\sqrt{N}} \sum_{k} \omega_{k} \mathrm{e}^{-\mathrm{i} \vec{k} \vec{l}}, \quad\left(\mathrm{~d} \omega_{k}\right)^{N}=\mathrm{d} \omega_{0} \prod_{k>0} \mathrm{~d} \omega_{k}^{c} \mathrm{~d} \omega_{k}^{s}=\sqrt{2}^{N-1} \prod_{l} \mathrm{~d} \omega_{l} .
\end{gathered}
$$

Cumulants $\tilde{\mathcal{M}}_{1616}$ and $\mathcal{M}_{16161616}$ depend on quasimomentum only by means of $\delta$ symbols [18], so, using the fourth-order basic measure density for $\rho_{k}$ :

$$
f(\omega)=\exp \left\{-\frac{(2 \pi)^{2}}{2} \tilde{\mathcal{M}}_{1616} \omega^{2}+\frac{(2 \pi)^{4}}{4 !} \mathcal{M}_{16161616} \omega^{4}\right\}
$$

after integrating over $\omega_{k}$ in (2.8), we obtain:

$$
Z=Z_{0} Z_{7} Z_{16}
$$

where

$$
Z_{16}=\sqrt{2}^{N-1} Q \int\left(\mathrm{d} \rho_{k}\right)^{N} \exp \left\{\frac{\beta}{2} \sum_{k} \Phi_{16}(\vec{k}) \rho_{k} \rho_{-k}\right\} \prod_{l}\left\{-\frac{1}{2} a_{2}^{(1)} \tilde{\rho}_{l}^{2}-\frac{1}{4 !} a_{4}^{(1)} \tilde{\rho}_{l}^{4}\right\} .
$$


Here

$$
\begin{aligned}
& \tilde{\rho}_{l}=\rho_{l}-\sqrt{N} \mathcal{M}_{16}, \quad Q=2 \int_{0}^{\infty} f(\omega) \mathrm{d} \omega \\
& a_{2}^{(1)}=(2 \pi)^{2} Q^{-1} \int_{-\infty}^{\infty} \omega^{2} f(\omega) \mathrm{d} \omega=-2 \frac{\partial}{\partial \tilde{\mathcal{M}}_{1616}} Q, \\
& a_{4}^{(1)}=-(2 \pi)^{4} Q^{-1} \int_{-\infty}^{\infty} \omega^{4} f(\omega) \mathrm{d} \omega+3 a_{2}^{2}=-4 Q^{-1} \frac{\partial^{2}}{\partial \tilde{\mathcal{M}}_{1616}^{2}} Q+3 a_{2}^{2} .
\end{aligned}
$$

The techniques of integrating the (3.5) form over the collective variables in the layers which contain $\rho_{\vec{k}}$ with a small deviation of $|\vec{k}|$ from a certain mean value, and the following transition to the point $\vec{k}=0$ was suggested for the isotropic Ising model in [17] and for the ferroelectric systems with dipole-dipole intercluster interactions in [21]. The essence of the method of integration proposed in [21] consists in the possibility to divide every layer of the Brillouin zone of the crystal into pairs of subzones, $B_{n}^{\mathrm{G}}$ and $B_{n}^{\mathrm{q}}$ according to the main types of fluctuation processes in each of them: Gaussian and non-Gaussian (quartic) ones. The sequence of such zones and subzones is determined by relations [21]:

$$
\begin{aligned}
B_{n}: \quad & {\left[0<|\vec{k}| \leqslant \frac{B_{1}}{s^{n-1}} ; \quad \theta_{n-1}<\theta \leqslant \pi-\theta_{n-1} ; \quad 0<\varphi \leqslant 2 \pi\right], } \\
B_{n}^{\mathrm{G}}: \quad & {\left[0<|\vec{k}| \leqslant \frac{B_{1}}{s^{n-1}} ; \quad \theta_{n-1}<\theta \leqslant \theta_{n}, \pi-\theta_{n}<\theta \leqslant \pi-\theta_{n-1} ; \quad 0<\varphi \leqslant 2 \pi\right], } \\
B_{n}^{\mathrm{q}}: \quad & {\left[\frac{B_{1}}{s^{n}}<|\vec{k}| \leqslant \frac{B_{1}}{s^{n-1}} ; \quad \theta_{n}<\theta \leqslant \pi-\theta_{n} ; \quad 0<\varphi \leqslant 2 \pi\right], }
\end{aligned}
$$

where $B_{1}$ is an initial Brillouin zone, $\theta$ and $\varphi$ are polar and azimuth angles in the ellipsoid of the rotating coordinate system, $s \geqslant 1$ is a parameter for dividing the Brillouin zone into layers [21],

$$
\theta_{n}=\arctan \sqrt{\frac{\beta \lambda}{\beta \varphi_{0}-a_{2}^{(n)}}-1} .
$$

After $(n-1)$ step of a two-stage layer-by-layer integration of (3.5) over $\rho_{k}$ variables for $Z_{16}$ we have the expression:

$$
\begin{aligned}
& Z_{16}=\sqrt{2}^{N_{1}} Q^{N} C_{0} \prod_{m=1}^{n-1}\{ \sqrt{2}^{-N_{m}} C_{m}\left[Q\left(\frac{d_{2}^{(m)}\left(\frac{B_{m}}{s}, B_{m}\right)}{(2 \pi)^{2}}, \frac{a_{4}^{(m)}}{(2 \pi)^{4}}\right)\right]^{N_{m}-N_{m}^{\mathrm{G}}} \\
& \times\left.\left.\times Q\left(P_{2}^{(m)}, P_{4}^{(m)}\right)\right]^{N_{m+1}}\right\} \\
& \times \int\left(\mathrm{d} \rho_{k}\right)^{N_{n}} \exp \left\{-\frac{1}{2} \sum_{k \in B_{n}} d_{2}^{(n)}(\vec{k}) \rho_{k} \rho_{-k}\right. \\
&\left.\quad-\frac{1}{4 ! N_{n}} \sum_{k_{1}, k_{2}, k_{3}, k_{4} \in B_{n}} a_{4}^{(n)} \rho_{k_{1}} \rho_{k_{2}} \rho_{k_{3}} \rho_{k_{4}} \delta\left(\vec{k}_{1}+\vec{k}_{2}+\vec{k}_{3}+\vec{k}_{4}\right)\right\}
\end{aligned}
$$


Here $Q\left(\frac{d_{2}^{(m)}}{(2 \pi)^{2}}, \frac{a_{4}^{(m)}}{(2 \pi)^{4}}\right), Q\left(P_{2}^{(m)}, P_{4}^{(m)}\right)$ are certain combinations of the modified Bessel functions of $z_{m}$ argument $[17,21]$ :

$$
\begin{aligned}
& z_{m}=\frac{3\left[d_{2}^{(m)}\left(\frac{B_{m}}{s}, B_{m}\right)\right]^{2}}{4 a_{4}^{(m)}} \\
& d_{2}^{(m)}\left(\frac{B_{m}}{s}, B_{m}\right)=\left\langle d_{2}^{(m)}(\vec{k})\right\rangle_{\frac{B_{m}}{s}, B_{m}}, \quad d_{2}^{(m)}(\vec{k})=a_{2}^{(m)}-\beta \Phi_{16}(\vec{k}), \\
& C_{m}=\prod_{k \in B_{m}^{\mathrm{G}}}\left\{\frac{\pi}{d_{2}^{(m)}(\vec{k})}\right\} \exp \left\{-\frac{a_{4}^{(m)}}{8 N} \sum_{k_{1}, k_{2} \in B_{m}^{\mathrm{G}}} \frac{1+\delta_{k_{1} k_{2}}}{d_{2}^{(m)}\left(\vec{k}_{1}\right) d_{2}^{(m)}\left(\vec{k}_{2}\right)}\right\}, \\
& P_{2}^{(m)}=\sqrt{\frac{12}{a_{4}^{(m)}}} K\left(z_{m}\right), \quad P_{4}^{(m)}=\frac{N_{m+1}}{N_{m}-N_{m}^{\mathrm{G}}} \frac{6}{a_{4}^{(m)}} L\left(z_{m}\right), \\
& K\left(z_{m}\right)=\sqrt{z_{m}}\left(\frac{K_{3 / 4}\left(z_{m}\right)}{K_{1 / 4}\left(z_{m}\right)}-1\right), \quad L\left(z_{m}\right)=6 K^{2}\left(z_{m}\right)+4 \sqrt{z_{m}} K\left(z_{m}\right)-1, \\
& N_{m}=\frac{N}{s^{3(m-1)}} \sqrt{\frac{1}{\beta \lambda}\left(\beta \varphi_{0}-a_{2}^{(m-1)}\right)}, \\
& N_{m}^{\mathrm{G}}=\frac{N}{s^{3(m-1)}}\left[\sqrt{\frac{1}{\beta \lambda}\left(\beta \varphi_{0}-a_{2}^{(m-1)}\right)}-\sqrt{\frac{1}{\beta \lambda}\left(\beta \varphi_{0}-a_{2}^{(m)}\right)}\right] .
\end{aligned}
$$

A general expression (3.9) is close to the one obtained in [20] for a cluster ferroelectric system. It should be underlined that the method of integration suggested here is based on taking into consideration the anisotropic character of the dipoledipole potential $\Phi_{16}(\vec{k})$, due to which the Gaussian coefficient $d_{2}^{(n)}(\vec{k})$ remains nonnegative in each subzone $B_{n}^{\mathrm{G}}$.

Coefficients $d_{2}^{(n)}(\vec{k})$ and $a_{4}^{(n)}$ determine the character of fluctuation processes in the cluster system near $T_{\mathrm{c}}$. Their mean values in different intervals of wave vectors (layers of integration) satisfy the following recursion relations [21]:

$$
\begin{aligned}
r_{n+1} & =s^{2}\left(r_{n}+q\right)\left\{\bar{N}_{n}+\frac{3}{4 z_{n}}\left(\bar{N}-\frac{1}{3}\right)\left(1-s^{-n} \sqrt{\frac{3}{2 \beta \lambda}\left(-r_{n}\right)}\right)\right\}-s^{2} q \\
u_{n+1} & =\sqrt{\frac{r_{n}}{r_{n-1}}} E_{n} U_{n} .
\end{aligned}
$$

Here

$$
\begin{array}{ll}
r_{n}=s^{2 n} d_{2}^{(n)}\left(\frac{B_{n}}{s}, B_{n}\right), & U_{n}=s^{4 n} a_{4}^{(n)}, \\
\bar{N}_{n}=\frac{2 \sqrt{\xi_{n}} K\left(\xi_{n}\right)}{3 \sqrt{z_{n}} K\left(z_{n}\right)}+\frac{1}{3}, & E_{n}=S^{6} \frac{L\left(\xi_{n}\right)}{L\left(z_{n}\right)}, \quad \xi_{n}=\frac{3}{2} s^{3} \frac{K^{2}\left(z_{n}\right)}{L\left(z_{n}\right)} .
\end{array}
$$

At $T<T_{\mathrm{c}}$, the integration over $\rho_{k}$ in (3.9) should be performed up to $n=\mu_{\tau}$, which is determined from the following relation:

$$
d_{2}^{\left(\mu_{\tau}\right)}\left(B_{\mu_{\tau}}\right)=0
$$


and it is equal to

$$
\mu_{\tau}=1+\ln \frac{c_{2} R-q}{c_{1}} / \ln E_{1} .
$$

Here, $E_{1}$ is the bigger of the two eigenvalues of the matrix of relations (3.11) linearized in the neighbourhood of its fixed point, $c_{1} \sim \tau, \tau=T_{\mathrm{c}}-T / T_{\mathrm{c}}, c_{2}, R, q$ are constants independent of $n$, (see [21]).

Assuming that the layer-by-layer integration in (3.9) has been performed up to the point $n=\mu_{\tau}$, let's go to the last stage of integration over $\rho_{k}$ with $|\vec{k}|<\left|B_{\mu_{\tau}}\right|$. From the definition of $\mu_{\tau}$ point (3.13) one can find that in the last layer of integration we have a Gaussian distribution for collective variables, but the coefficients of this distribution are nonanalytical. Such a specific Gaussian distribution is called the inverse Gaussian distribution [17].

The correct extraction of collective variables with zero values of quasimomentum needs to take into account the displacement of the center of fluctuation of $\rho_{k}$ in the ferromagnetic phase (at a nonequal to zero external field in paramagnetic phase as well):

$$
\rho_{k}=\rho_{k^{\prime}}+\sqrt{N}\langle\sigma\rangle \delta(\vec{k}) .
$$

Such a procedure for a similar functional concerning a ferroelectric phase transition is described in detail in [20]. With the accuracy up to constant terms, the results from [20] may be reproduced here. Hence, the fourth-order form integrand in (3.9) for the last $\left(n=\mu_{\tau}\right)$ stage of integration transforms into

$$
E_{\mu_{\tau}}\left(\rho_{0}\right)=\left[a_{2}^{\left(\mu_{\tau}\right)}+2 \bar{d}_{2}(0)\right] \sqrt{N} \mathcal{M}_{16} \rho_{0}+\frac{1}{4}\left[\bar{d}_{2}(0)-a_{4}^{\left(\mu_{\tau}\right)} I\right] \rho_{0}^{2}-\frac{1}{N} a_{4}^{\left(\mu_{\tau}\right)} \rho_{0}^{4},
$$

where

$$
\bar{d}_{2}(\vec{k})=2\left|d_{2}^{\left(\mu_{\tau}\right)}(0)\right|+q k^{2}, \quad I=\frac{1}{N_{\mu_{\tau}}} \sum_{k} \frac{1}{\bar{d}_{2}(\vec{k})} .
$$

For spherical-symmetric potential $\Phi(\vec{k})$

$$
I=\frac{3}{\bar{d}_{2}(0)} \frac{t-\arctan t}{t^{3}}, \quad t=\frac{\pi}{b s^{\mu_{\tau}}} \sqrt{\frac{q}{d_{2}(0)}},
$$

$b$ is a lattice constant [17].

A similar expression may be also obtained for $\Phi_{16}(\vec{k})(1.3)$. However, taking into account that at $n=\mu_{\tau}$ the polar angle $\theta \approx \pi / 2$, the expression (3.18) in this case also holds.

Finally, for functional integral in (3.9) we have obtained an expression:

$$
Z_{\mu_{\tau}}=f(T) \int \exp \left\{-N\left[D \rho^{4}-B \rho^{2}-A \rho\right]\right\} \mathrm{d} \rho,
$$

where $f(T)$ is a continuous function of temperature in the $T_{\mathrm{c}}$ neighbourhood,

$$
A=\left[a_{2}^{\left(\mu_{\tau}\right)}+\frac{1}{2} \bar{d}_{2}(0)\right] \mathcal{M}_{16}, \quad B=\frac{1}{4}\left[\bar{d}_{2}(0)-a_{4}^{\left(\mu_{\tau}\right)} I\right], \quad D=\frac{1}{4 !} \frac{N}{N_{\mu_{\tau}}} a_{4}^{\left(\mu_{\tau}\right)} .
$$


Thus, the procedure of integrating the partition function functional over collective variables is reduced to the simple one-multiple integral (3.19). The critical characteristics of the system investigated are described by $Z_{\mu_{\tau}}$.

\section{Equation for order parameter of the cluster magnetics}

The equation for the order parameter, i.e., the equation of state, of the cluster magnetics near a ferromagnetic phase transition point has been found after minimizing the integrand in (3.19) with respect to the variable $\rho$ :

$$
\frac{\partial}{\partial \rho}\left(D \rho^{4}-B \rho^{2}-A \rho\right)=0
$$

or

$$
4 D \rho^{3}-2 B \rho-A=0 .
$$

To investigate the properties of (4.2) and to find its solutions, the detailed dependencies of coefficients $A, B, D$ under temperature $T$ and at an external field $h$ must be determined. Solutions $d_{2}^{(m)}(0), a_{4}^{(m)}$ for a linearized system of recursion equations (3.11) in the renormalized perturbation theory approximation were obtained in [20]. These solutions are general for Hamiltonians with dipole-dipole intercluster interaction, and so are the ones considered here. We have:

$$
\begin{array}{ll}
d_{2}^{\left(\mu_{\tau}\right)}(0)=\frac{c_{1}^{\prime} E_{1}^{\mu_{\tau}-1}-c_{2} R}{s^{2\left(\mu_{\tau}-1\right)}}, & \bar{d}_{2}(0)=2\left|d_{2}^{\left(\mu_{\tau}\right)}(0)\right|, \quad a_{4}^{\left(\mu_{\tau}\right)}=\frac{c_{1}^{\prime} R^{\prime} E_{1}^{\mu_{\tau}-1}+c_{2}}{s^{4\left(\mu_{\tau}-1\right)}} \\
s^{-2\left(\mu_{\tau}-1\right)}=\frac{c_{1}^{\prime}}{c_{2} R-\beta \Phi_{16}(0)}, & E_{1} \sim s^{2}, \quad c_{1}^{\prime}=\tilde{c}_{1} \tau \ln ^{-1 / 3}|\tau|, \quad \tilde{c}_{1}=\frac{3 \beta \Phi_{16}(0)}{\left(3^{4} \gamma\right)^{1 / 3}}
\end{array}
$$

$\tilde{c}_{1}, R, R^{\prime}, \gamma$ are independent of $\tau$ constants [20].

Taking into account that $\tau$ changes its sign when $T$ passes to $T_{\mathrm{c}}$, we present $\bar{d}_{2}(0)$ and $a_{4}^{\left(\mu_{\tau}\right)}$ as follows:

$$
\begin{aligned}
\bar{d}_{2}(0) & =\frac{2 \beta \Phi_{16}(0) \tilde{c}_{1}}{c_{2} R-\beta \Phi_{16}(0)} \tau \ln ^{-1 / 3}|\tau|=\Delta_{1} \tau \ln ^{-1 / 3}|\tau| \\
a_{4}^{\left(\mu_{\tau}\right)} & =\frac{\left(c_{2}\left(R R^{\prime}+1\right)-\beta \Phi_{16}(0) R^{\prime}\right) \tilde{c}_{1}^{2}}{\left(c_{2} R-\beta \Phi(0)\right)^{2}} \tau^{2} \ln ^{-2 / 3}|\tau|=\Delta_{2} \tau^{2} \ln ^{-2 / 3}|\tau|
\end{aligned}
$$

For the coefficients of (4.2) equation we have:

$$
\begin{aligned}
& A=\left[\beta \Phi_{16}(0)+\Delta_{1} \tau \ln ^{-1 / 3}|\tau|\right] \frac{\sqrt{2} \sinh \beta h}{1+2 \cosh \beta h+\mathrm{e}^{-2 \beta V}} \\
& B=\frac{\Delta_{1}}{4}\left(1-\frac{3 \Delta_{2}}{\Delta_{1}} \frac{t-\arctan t}{t^{3}}\right) \tau \ln ^{-1 / 3}|\tau| \\
& D=\frac{c_{2}^{2} R^{2} \Delta_{2}}{3 ! \Delta_{1}^{2}} \ln ^{-2 / 3}|\tau|
\end{aligned}
$$


At small values of the external magnetic field $h$ it is convenient to present equation (4.2) in the form:

$$
\rho^{3}-v \tau \ln ^{1 / 3}|\tau| \rho-\left(w_{1} \tau \ln ^{1 / 3}|\tau|+w_{2} \ln ^{2 / 3}|\tau|\right) \beta h=0
$$

where

$$
\begin{aligned}
& v=3 \Delta_{1}\left(\Delta_{1}^{2}-3 \Delta_{2} \frac{t-\arctan t}{t^{3}}\right) / 4 c_{2}^{2} R^{2} \Delta_{2}, \\
& w_{1}=\frac{3 \Delta_{1}^{2}}{\sqrt{2} c_{2}^{2} R^{2} \Delta_{2}\left(3+\mathrm{e}^{-2 \beta V}\right)}, \quad w_{2}=\frac{3 \Delta_{1}^{3} \beta \Phi(0)}{\sqrt{2} c_{2}^{2} R^{2} \Delta_{2}\left(3+\mathrm{e}^{-2 \beta V}\right)} .
\end{aligned}
$$

Thus, we have obtained the equation for the order parameter (i.e., the equation of state) of a magnetic cluster system with dipole-dipole intercluster interaction, which is valuable in the ferromagnetic phase transition neighbourhood. All the coefficients of this equation are determined in a microscopic way, which depends only on initial Hamiltonian parameters, on temperature and on the external magnetic field. Equation (4.6), (4.7) is close in form to the equation of state for a ferroelectric cluster system [20], but distinguishes by another shape of coefficients $\left(w_{1}\right.$ and $w_{2}$ instead of only $w$ in [20]) and by their different physical sense.

According to Cardanault formula [22], solutions of (4.6) may be presented in the form:

$$
\rho_{1}=L+M, \quad \rho_{2,3}=-\frac{L+M}{2} \pm \mathrm{i} \sqrt{3} \frac{L-M}{2},
$$

where

$$
\begin{aligned}
& L=\sqrt{3\left(w_{1} \tau \ln ^{1 / 3}|\tau|+w_{2} \ln ^{2 / 3}|\tau|\right) \beta h+\sqrt{N}} \\
& M=\sqrt{3\left(w_{1} \tau \ln ^{1 / 3}|\tau|+w_{2} \ln ^{2 / 3}|\tau|\right) \beta h-\sqrt{N}} \\
& N=-\left(\frac{v}{3}\right)^{3} \tau^{3} \ln |\tau|+\left(w_{1} \tau \ln ^{1 / 3}|\tau|+w_{2} \ln ^{2 / 3}|\tau|\right)^{2} \frac{\beta^{2} h^{2}}{4} .
\end{aligned}
$$

There are three different regimes in the roots (4.8) behaviour at $T<T_{\mathrm{c}}$ and two regimes at $T>T_{\mathrm{c}}$.

At $T<T_{\mathrm{c}}$ :

1) $h=0, R<0$

$$
\rho_{1,2}= \pm v^{1 / 2}|\tau|^{1 / 2} \ln ^{1 / 6}|\tau|, \quad \rho_{3}=0 ;
$$

2) $h \neq 0, R<0$ (small value of $h$ ), there are 3 different non-equal to zero roots;

3) $h \neq 0, R>0$ (large value of $h$ ),

$$
\rho=\left(w_{1} \tau \ln ^{1 / 3}|\tau|+w_{2} \ln ^{2 / 3}|\tau|\right)^{1 / 3}(\beta h)^{1 / 3}
$$

and two compositely conjugated roots. 
At $T>T_{\mathrm{c}}$ :

1) $h=0, R>0$

$$
\rho=0
$$

and two compositely conjugated roots,

2) $h \neq 0, R>0$

$$
\rho=\left(w_{1} \tau \ln ^{1 / 3}|\tau|+w_{2} \ln ^{2 / 3}|\tau|\right)^{1 / 3}(\beta h)^{1 / 3}
$$

and two compositely conjugated roots.

The precise analysis of the order parameter (or the spontaneous magnetization proportional to it) near $T_{\mathrm{c}}$ may be done using the derivations $\partial \rho / \partial \tau$ and $\partial \rho / \partial \beta h$. Based on the equation (4.6) we have obtained:

at $h=0$ :

$$
\frac{\partial \rho}{\partial \tau}=\frac{v^{1 / 2} \ln ^{1 / 6}|\tau|}{2 \tau^{1 / 2}}, \quad \frac{\partial \rho}{\partial \beta h}=\frac{w_{2} \ln ^{1 / 3}|\tau|}{2 v \tau}
$$

at $h \neq 0$ :

$$
\begin{aligned}
& \frac{\partial \rho}{\partial \tau}=\frac{v \rho\left[w_{1}+\frac{2}{3} w_{2} \tau^{-1} \ln ^{-2 / 3}|\tau|\right] \beta h}{3 \rho^{2} \ln ^{-1 / 3}-v \tau} \approx \frac{2 w_{2} \beta h}{9 \rho^{2} \tau \ln ^{1 / 3}|\tau|} \\
& \frac{\partial \rho}{\partial \tau}=\frac{w_{1} \tau+w_{2} \ln ^{1 / 3}|\tau|}{3 \rho^{2} \ln ^{-1 / 3}-v \tau} \approx \frac{w_{2} \ln ^{3 / 2}|\tau|}{3 \rho^{2}}
\end{aligned}
$$

The temperature dependence of the real roots of equation (4.6) at different values of the external field is presented in figure 1 . We can see that near $\tau=0\left(T \approx T_{\mathrm{c}}\right)$ and $h \neq 0 \rho(\tau)$ has got a bend which is rather smoothed by the logarithmic corrections. Just as at $h=0$, such a bend is absent and $\rho(\tau)$ demonstrates a sharper behaviour. The field dependence of $\rho(h)$ (figure 2 for $T<T_{\mathrm{c}}$ and figure 3 for $T>T_{\mathrm{c}}$ ) shows a similar tendency. This phenomenon is a reason for the "field softening" of magnetic susceptibility at $\tau=0$ [23].

Near $T_{\mathrm{c}}$ (small $\tau$ ), a static magnetic susceptibility of cluster ferromagnets may be obtained in the form:

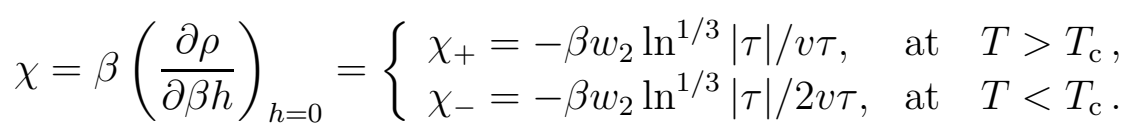

The width of the region for an abnormal increase of $\chi$ near $T_{\mathrm{c}}$ is smaller with large $V$ (i.e., energy of intercluster interaction between particles). Divergency of $\chi$ is weaker as compared with Ising model [24]. The "law of doubling" $\left(\chi_{+}=2 \chi_{-}\right)$has been fulfilled.

The main difference in the critical dependence of the order parameter obtained here and presented in [25] is a correction $\ln ^{1 / 6}|\tau|$ instead of $\ln ^{1 / 3}|\tau|$. In [25] the simple perturbation procedure for the selected types of diagrams based on renormalized Gaussian integrals was used. After summing up an infinite series of renormalized 


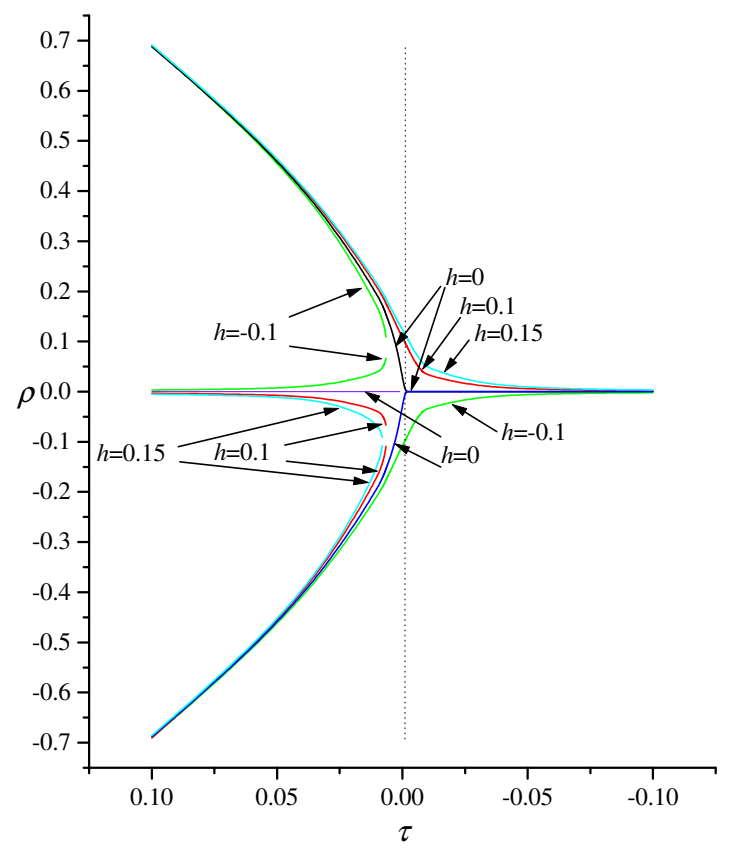

Figure 1. Temperature dependencies of the roots of equation (4.6) for cluster magnetics in the phase transition point neighbourhood at different values of the external field $h$.

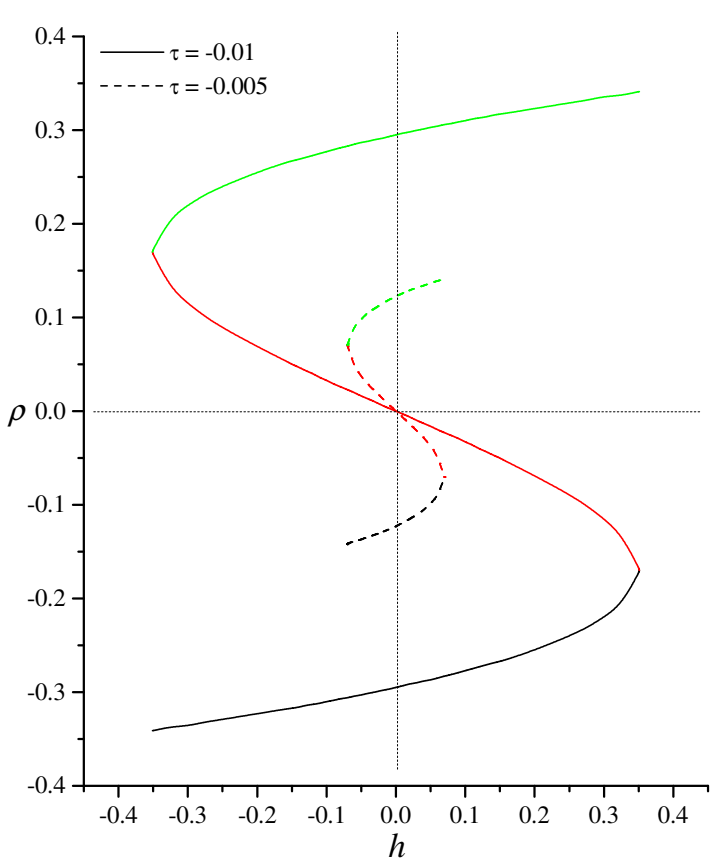

Figure 2. Field dependencies of real roots of equation (4.6) for cluster magnetics at $T<T_{\mathrm{c}}$.

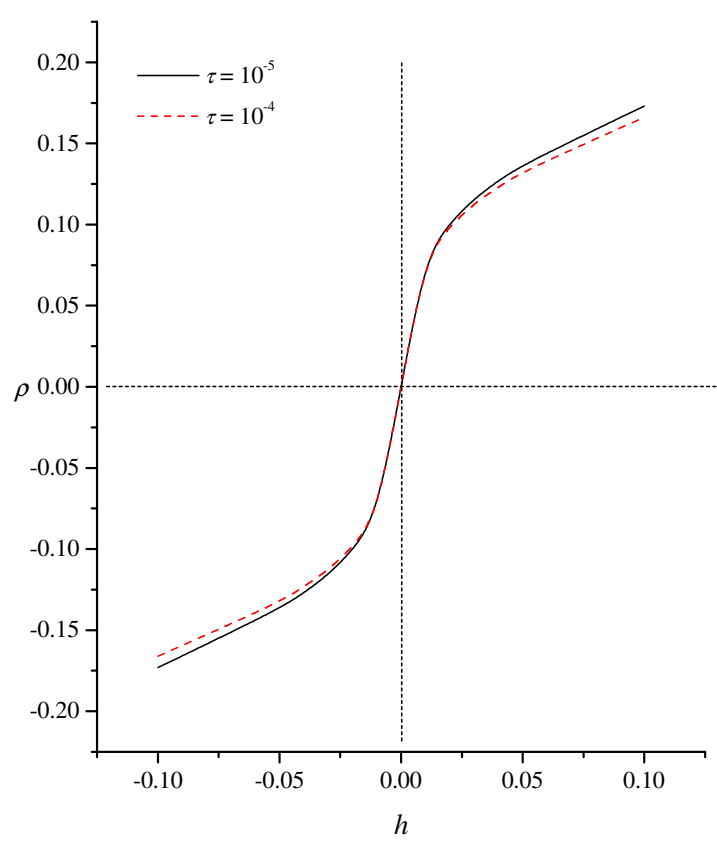

Figure 3. Field dependencies of real roots of equation (4.6) for cluster magnetics at $T>T_{\mathrm{c}}$. 
Gaussian integrals, the effective exponent in logarithmic correction may be reduced. Due to using here a non-Gaussian basic measure density for collective variables in the phase transition point neighbourhood, the exponent at logarithmic correction $1 / 6$ is obtained at once.

Introducing "effective critical exponents" $\beta_{1 / 6}$ and $\beta_{1 / 3}$ for both cases:

$$
\rho= \pm v^{1 / 2} \tau^{1 / 2} \ln ^{1 / 6}|\tau|= \pm v^{1 / 2} \tau^{\beta_{1 / 6}}, \quad \rho= \pm v^{1 / 2} \tau^{1 / 2} \ln ^{1 / 3}|\tau|= \pm v^{1 / 2} \tau^{\beta_{1 / 3}}
$$

at different $\tau$ we have

\begin{tabular}{|l|l|l|l|l|l|l|l|l|l|}
\hline$\tau$ & $10^{-1}$ & $5 \cdot 10^{-2}$ & $10^{-2}$ & $10^{-4}$ & $10^{-6}$ & $10^{-8}$ & $10^{-10}$ & $10^{-12}$ & $10^{-20}$ \\
\hline$\beta_{1 / 3}$ & 0.3793 & 0.3779 & 0.3894 & 0.4247 & 0.4366 & 0.4473 & 0.4546 & 0.460 & 0.4723 \\
\hline$\beta_{1 / 6}$ & 0.4396 & 0.4390 & 0.4447 & 0.4598 & 0.4683 & 0.4736 & 0.4773 & 0.480 & 0.4861 \\
\hline
\end{tabular}

It is well-known that the critical behaviour for most ferromagnetics and ferroelectrics is observed in the temperature region $\tau^{*} \approx 0,1$ [24]. Thus, it may be considered that at $\tau \approx 0.1$, the "effective critical exponent" $\beta$ should be close to $\beta_{k l}=0.5$. As we see in the table, this requirement is better to satisfy by the exponent $\beta_{1 / 6}$ than $\beta_{1 / 3}$. Hence, the power $1 / 6$ in logarithmic corrections is more adequate to a real situation in the dipole magnetic systems.

\section{References}

1. Weiss P. // J. phys. et radium, 1907, vol. 6, p. 661.

2. Smart J.S. Effective Field Theories of Magnetism. Philadelphia-London, W.B.Saunders comp., 1966.

3. Blinc R., Svetina S. // Phys. Rev., 1966, vol. 147, p. 423.

4. Kubo R. // J. Phys. Soc. Japan, 1962, vol. 17, p. 1100.

5. Vaks V.G. Introduction to Microscopic Theory of Ferroelectrics. Moskow, Nauka, 1973 (in Russian).

6. Levitskii R.R., Korynevskii N.A., Stasyuk I.V. // Ukr. Phys. J., 1974, vol. 19, p. 1289 (in Russian).

7. Levitskii R.R., Korynevskii N.A., Stasyuk I.V. // Phys. Stat. Sol. (b), 1978, vol. 81, p. 51 .

8. Korynevskii N.A., Levitskii R.R. // Theor. Mat. Phys., 1980, vol. 42, p. 416 (in Russian).

9. Levitskii R.R., Grigas J., Zachek I.R., Mits Ye.V., Paprotny W. // Ferroelectrics, 1986, vol. 67, p. 109.

10. Yukhnovskii I.R., Levitskii R.R., Sorokov S.I. Theory of quasispin systems with the basis account of short-range interactions. - Modern Problems of Statistical Physics. Proceedings of All-Union Conference. Kiev, Naukova dumka, 1989, vol. 1, p. 392 (in Russian).

11. Levitskii R.R., Sorokov S.I. // Condens. Matter Phys., 1994, No. 3, p. 79.

12. Levitskii R.R., Sorokov S.I., Baran O.R., Pyndzyn I.M. // Journ. Phys. Stud., 1998, vol. 2. p. 391.

13. Baran O.R., Levitskii R.R. // Phys. Stat. Sol. (b), 2000, vol. 219, p. 357. 
14. Stasyuk I.V., Levitskii R.R., Zachek I.R., Moina A.P. // Phys. Rev. B, 2000, vol. 62, p. 6198.

15. Stasyuk I.V., Levitskii R.R., Moina A.P., Lisnii B.M. // Ferroelectrics, 2001, vol. 254, p. 213.

16. Baran O.R., Levitskii R.R. // Phys. Rev. B, 2002, vol. 65, p. 172407.

17. Yukhnovskii I.R. Phase Transition of the Second Order. Collective Variables Method. Singapore, World Scientific, 1986.

18. Korynevskii N.A. // Condens. Matter Phys., 2002, vol. 5 , No. 3(31), p. 391-412.

19. Born M., Huang K. Dynamic Theory of Crystalline Lattices. Moskow. Izd. inostr. lit., 1958 (in Russian).

20. Korynevskii N.A. // Condens. Matter Phys., 1999, vol. 2, No. 4(20), p. 631.

21. Yukhnovskii I.R., Korynevskii N.A. // Phys. Stat. Sol. (b), 1989, vol. 154, p. 519.

22. Korn G.A., Korn T.M. Mathematical Handbook for Scientists and Engineers. McGraw-Hill Book Company, 1961.

23. Landau L.D., Lifshits E.M. Statistical Physics. Vol. 1. Moskow, Nauka, 1976 (in Russian).

24. Yukhnovskii I.R., Kozlovskii M.P., Pylyuk I.V. Microscopic Theory of the Phase Transitions in Three-dimensions systems. Lviv, Evrosvit, 2001 (in Ukrainian).

25. Larkin A.I., Khmelnitskii D.E. // JETF, 1969, vol. 56, p. 2087 (in Russian).

\section{Параметр порядку феромагнітного фазового переходу в магнітній системі двочастинкових кластерів}

\section{М.А.Кориневський ${ }^{1,2}$}

1 Інститут фізики конденсованих систем НАН України, 79011 Львів, вул. Свєнціцького, 1

2 Інститут фізики Щецінського університету, Польща, 70-451 Щецін, вул. Вєлькопольська, 15

Отримано 18 вересня 2002 p.

З використанням четверної міри виконано інтегрування за колективними змінними у функціоналі статистичної суми магнітної системи двочастинкових кластерів. Для правильного врахування гаусових i негаусових флуктуацій параметра порядку у випадку диполь-дипольних міжкластерних взаємодій використано метод пошарового інтегрування. Для отримання рівняння для параметра впорядкування застосовано мінімізаційну процедуру підінтегрального виразу відносно останньої непроінтегрованої колективної змінної, яка відповідає нульовим значенням квазіімпульсу та мацубарівської частоти. Знайдено розв' язок цього рівняння в околі точки фазового переходу.

Ключові слова: феромагнетики, кластерні системи, функціонал, параметр впорядкування

PACS: $05.60 .+W, 05.70 . \mathrm{Ln}, 05.20 . \mathrm{Dd}, 52.25 . \mathrm{Dg}, 52.25 . \mathrm{Fi}$ 